\title{
of Mathematical models for predicating draft forces of tillage tools: A Review
}

\section{النماذج الرياضية لتوقع قوى الثد لأسلحة الحرث: دراسة مرجعية}

Since 1996

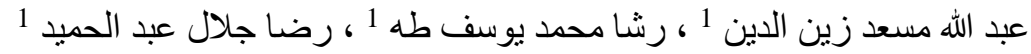

$$
\begin{aligned}
& 1 \text { قسم الهندسة الزراعية والنظم الحيوية - كلية الزراعة الثاطبي - جامعة الإسكندرية - جمهورية مصر العربية. }
\end{aligned}
$$

DOI: $10.21608 /$ jalexu.2021.170698

\section{Article Information}

Received:January $21^{\text {th }} 2021$

Revised: January $30^{\text {th }} 2021$

Accepted:February $11^{\text {th }} 2021$

Published: May $11^{\text {th }} 2021$
ENGLISH SUMMARY: tillage play an important aspect in the evolution of agriculture. Also, it has a direct influence on soil productivity and sustainability. The function of the tillage includes seedbed preparation, water and soil conservation and weed control. A large amount of energy is consumed during tillage operations. Furthermore, the draft force results in frictional and wear losses of the tillage equipment. Since the cost of energy is high and there are significant energy losses due to the friction and wear, the development of highly efficient tillage tools that require less energy while providing a satisfactory soil environment for seed emergence is of paramount importance . There is a lack of knowledge in soil conditions required for crop, soil dynamics to apply energy to the soil, to change its state from the original to the desired state and development of control algorithms and tillage transducers. With increased use of computers, it is possible to model the entire machine- soil plant system in order to make the analysis of tillage a more deterministic rather than the present stochastic process. To achieve this goal, the system must be divided into soil - machine sub-model and soil-plant sub model. Draft force prediction, clod sizes distribution and negative effect of erosion have been some of the motivation topics for studying and modeling soil-tillage interaction. Data from field process and lab experiments are combined on mathematical models; supporting by the increment on informatics resources more accurate, fasters and extended prognostics are performed (Bravo et al., 2012). These models can be classified as:

1-Experimental models.

2 -Analytical models.

3 -Numerical model (Finite Element Models).

On the other hand, Dimensional Analysis Models, and statistical models were also included.in order to recommend one model on the other, it was decided to compare their results with some experimental tests. Some of the models were succeeded to predict the draft in a range of 40-60\%, And they fail in some other conditions. Wherever the FEM give prediction up to 80-90\% accurate .

Any development in that direction will be rewarded, where it represents the real situation during loading, Where the close relation between soil and tool is thoroughly considered.

Keywords: Mathematical models, draft forces, tillage tools.

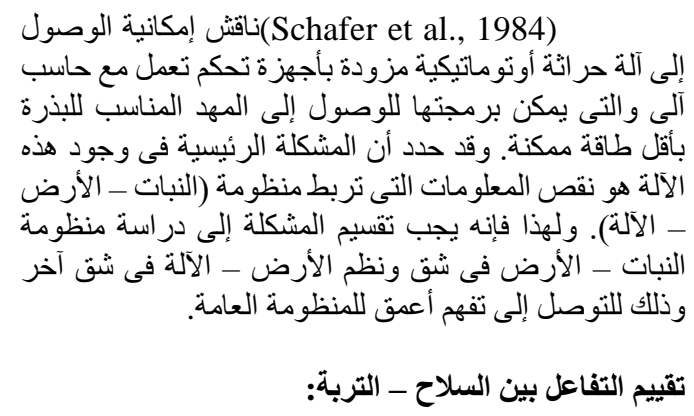


للوصول إلى وصف عام رياضى لتأثير بعض العو امل الهامة

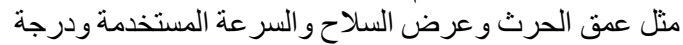

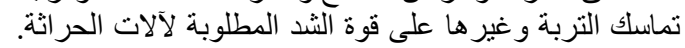

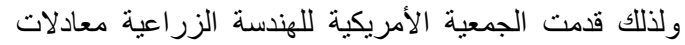

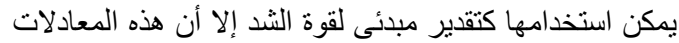

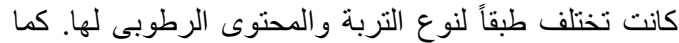

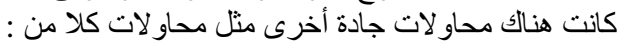

‘(Bowers, 1985)،(Upadhyaya, 1984)

(Yasin et al., 1991), ‘(Nicholson et al., 1984)

(Oni et al., 1992),(Grisso et al., 1996). ومن الجدير بالذكر أن كل هذه النماذج لم تدخل فى النى

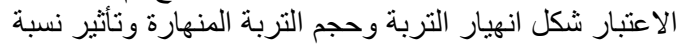

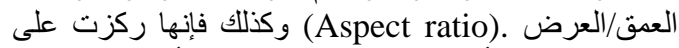

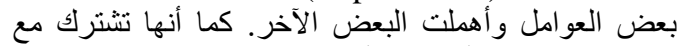

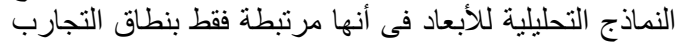

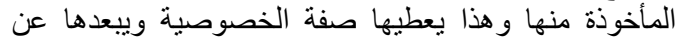
كونها معادلات عامة للاستخدام.

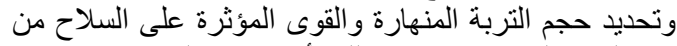

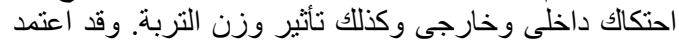

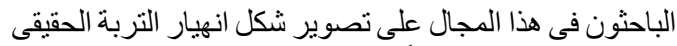

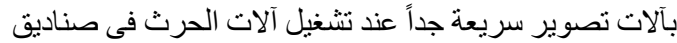
التربة الزجاجية وكذللك الاستعانة بوسائل أخرى لتهن لتحديد

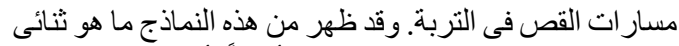

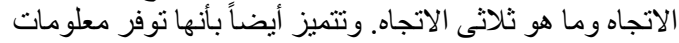

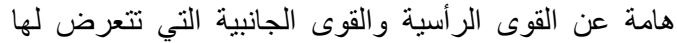

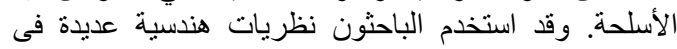

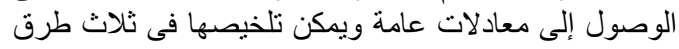

1- $\quad$ Trial wedge method

2- $\quad$ Stress characteristic method

3- Critical state soil mechanics models

\section{1- Trial wedge method}

وتعتمد هذه الطريقة على Rankines theory التى الم

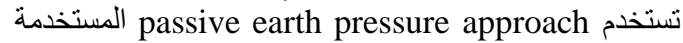
فى الهندسة المدنية فى تحليل القوى فى الحوائط الساندة

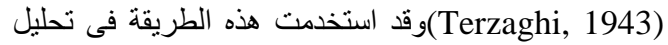
نظام السلاح - التربة حيث مثل السلاح بالحو ائط الساندة.

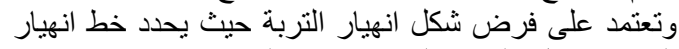

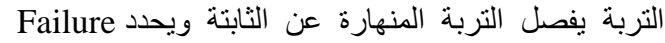
بboundary.

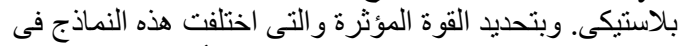
فرضهاو عمل اتز ان لهذه القوى مع استخدام أى طريقة رياضية To minimize forces.」 ومن الجدير بالذكر أن هناك العديد من النماذج

التحليلية قد ظهرت حتى الآونة الأخيرة إلا أنها تختلف فئل فيما بينها

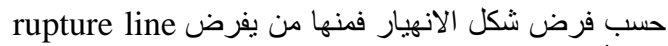

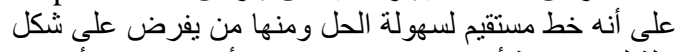

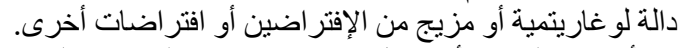

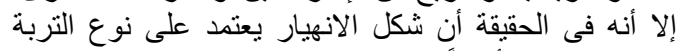

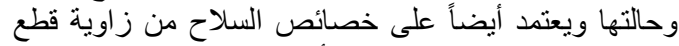

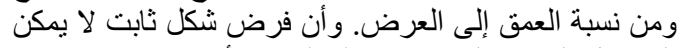

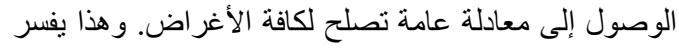

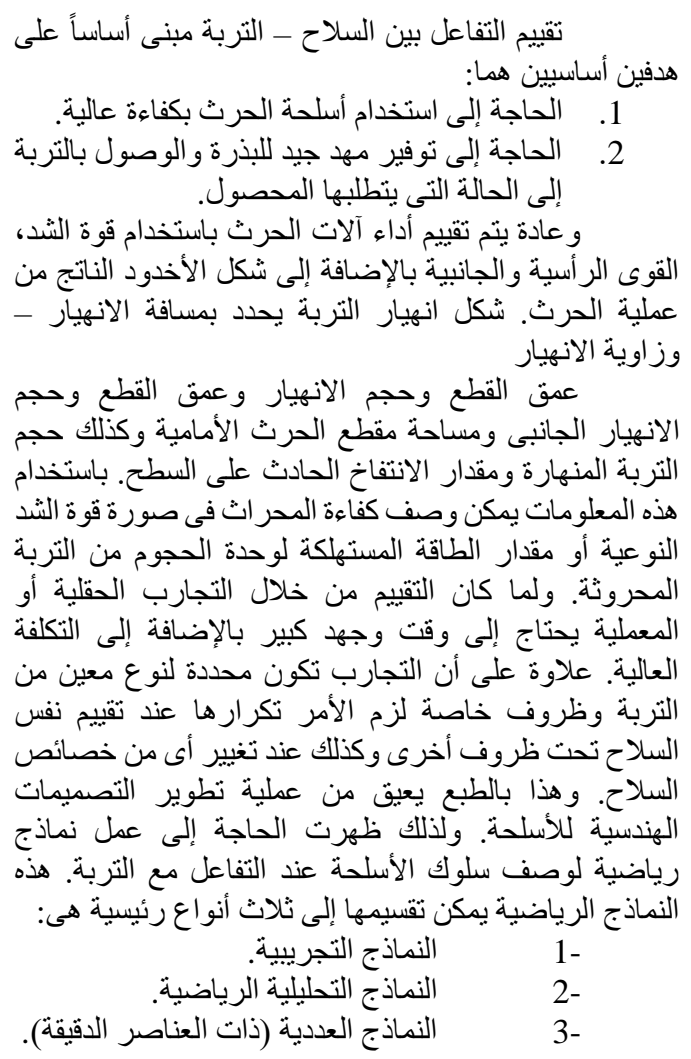

(Drwish, 2020).

حدد (Young, 1968) أنه بمكن استخدام التحليل البعدى فى دراسة التفاعل بين أسلحة الحرث والنه والتربة كما أنه النه

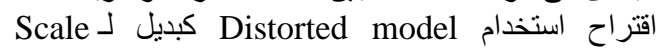
models الطبيعية و الميكانيكية. ولكنه أيضاً أكد عدم دقة هذه النماذج فيى فئ هذا وقد بدأ (Osman, 1964) في تقديم معادلات توقع شدة الثد.

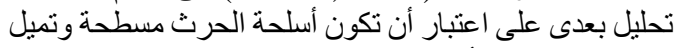

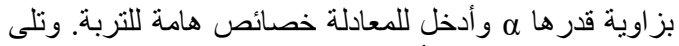

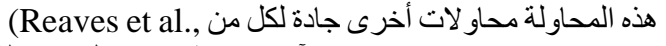

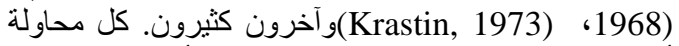

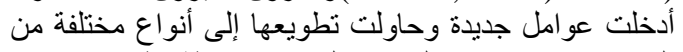

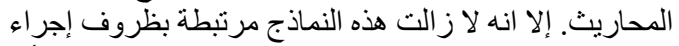

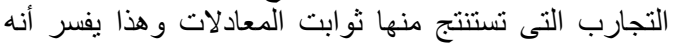

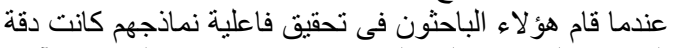

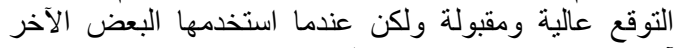

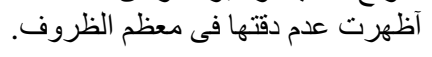

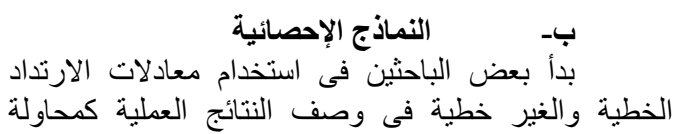




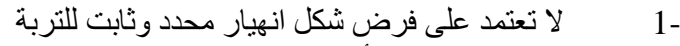

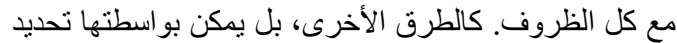

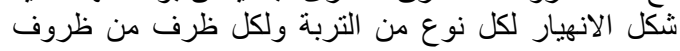

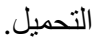

2-

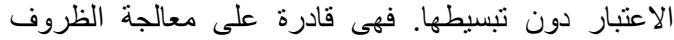
المحيطة المعقدة.

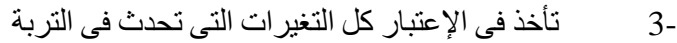

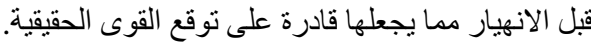

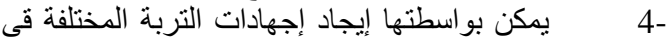

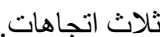

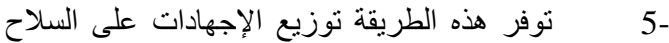

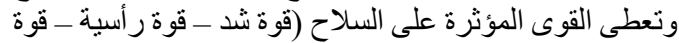
جانبية).

وتعتمد هذه الطريقة على تقسيم نظام السلاح ـ التربة

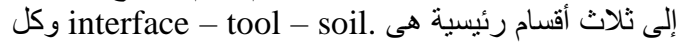

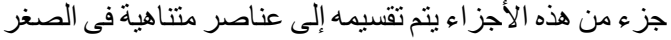

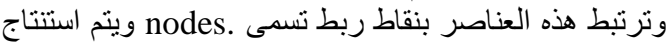

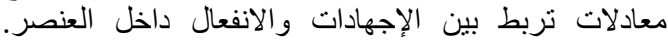

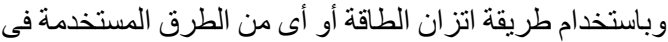

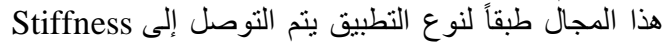

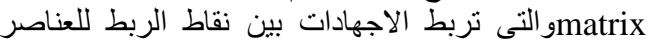

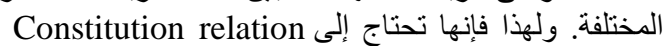

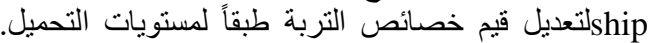

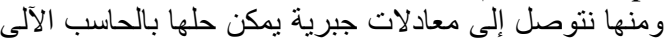

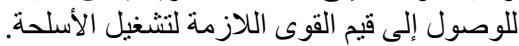
وقد ظهرت في الآونة الأخيرة نماذج الأنة عديدة تعتمد على FEM منها

(Constantin et al., 2019; Fielke, 1999;

Plouffe et al., 1999; Yong \& Hanna, 1977; Zein Eldin et al., 1990; Zhang et al., 2020)

وبر غم المميز ات العديدة التى تتميز بها هذه الطريقة

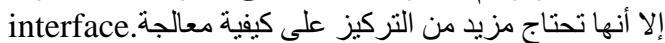
نتائج مقارنة أهم النماذج الرياضية باذية بالنتائج العملية:

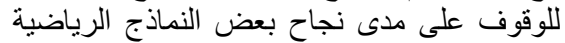

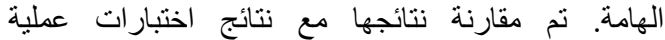

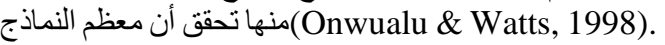

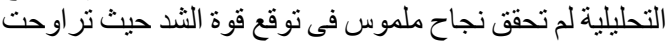

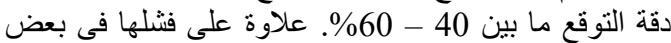

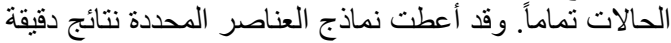

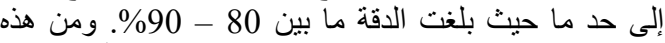

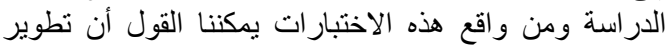

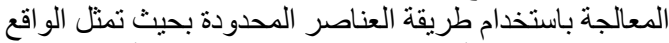

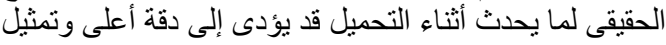

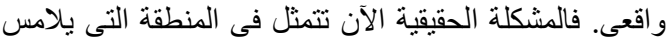

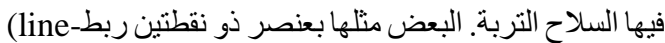

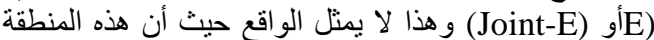

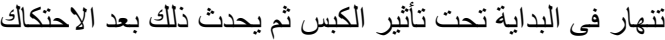

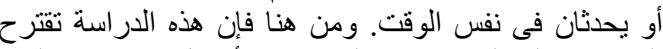
الميزد من المعالجة فى هذه الجزئية فى أُعمال بحثية مستقبلة.

\section{REFERENCES}

Bowers, C. G. (1985). Southeastern tillage energy data and recommended reporting. Transactions of the ASAE, 28(3), 731-737.

Bravo, E. L., Suárez, M. H., Cueto, O. G.,
السبب فى عدم دقة هذه النماذج فى معظم الدراسات التى هدفت لتحقيق فاعليتها (Onwualu \& Watts, 1998) و غير هي هم. وقد تضمنت هذه الدراسة المرجعية دراسة لأهم الهم

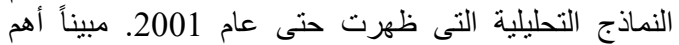
الفروض لكل منها ومميزات وقصور كل منها. و هذه النماذج

(Godwin \& Spoor, 1977; Hettiaratchi \& Reece, 1967; E McKyes \& Ali, 1977; O'callaghan \& Farrelly, 1964; Payne, 1956; Perumpral et al., 1983; Swick \& Perumpral, 1988; Zein El-Din \& Sayedahmed, 2000)

\section{2- $\quad$ Stress characteristic model}

معظم نماذج Wedge approach تعتمد على

محاو لات حسابية To minimize the tools forces ولذانلك فإن محاولة الوصول إلى شكل انهيار التربة الحقيقى بدون

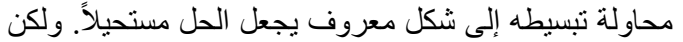

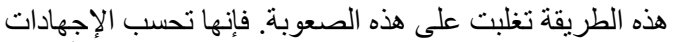

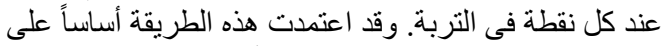
Mohr-Coulomb failure. التربة أيضاً إلا أنها لا تعامل كتلة التربة المنهارة كأنها وحدة أنهار

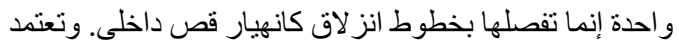

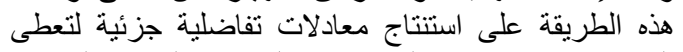

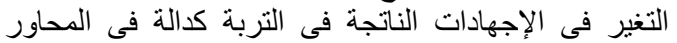

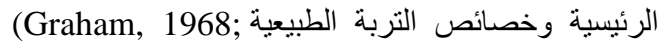
Edward McKyes, 1985) الطرق العددية لحل هذه المعادلات.

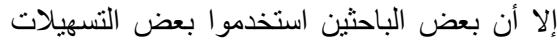

الفرضية متل فرض أن خطوط الانز لاق خطوط مستقيمة وذلك الكي

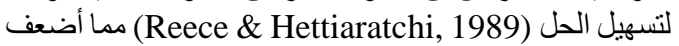
دقة توقعها. كما أن اعتمادها على نطرية Mohr حال دون

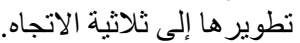

\section{3- Critical state soil mechanics models}

بالنظر إلى جميع الطرق السابق مناقثتها نجد أنها

تفرض أن كتلة التربة تحت اتز ان بلاستيكى و أنه لا يو جد تشكل

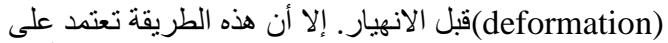

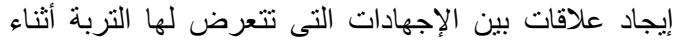

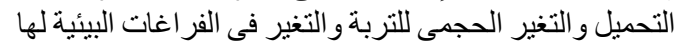

(Kawamura, 1985).

ومن الجدير بالذكر أن هذه الطريقة رغم أنها ثلاثية الاتجاه فى طبيعتها إلا أنها لم تحظى بقان الطدر كبير من الاستخدام

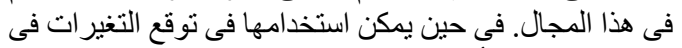

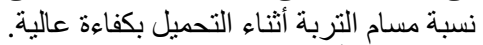
ثالثأ: النماذج العددية (النماذج ذات العناصة الثناء الدقيقة): بدأ استخدام طريقة العناصر المحدودة فى تطبيقات العنات

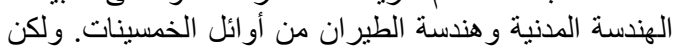

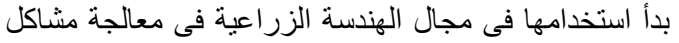

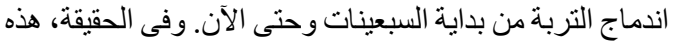

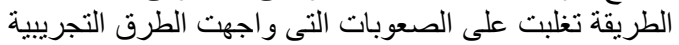
والطرق التحليلية فهى:

Tijskens, E., \& Ramon, H. (2012). Numerical Simulation of Soil-Tool Interaction by Discrete Element Method. Revista Ciencias Técnicas Agropecuarias, 21(4), 5-11.

Constantin, G. A., Voicu, G., Olac, B., Ilie, F., \& 
Paraschiv, G. (2019). Structural analysis with finite elements of a subsoiler working part. International Symposium, ISB-INMATEH, Agricultural and Mechanical Engineering, Bucharest, Romania, 31 October-1 November 2019., 89-95.

Drwish, L. A. (2020). Modeling the Effect of SoilTool Interaction on Draft Force Using Visual Basic. Annals of Agricultural Science, Moshtohor, 58(2), 223-232.

Fielke, J. M. (1999). Finite element modelling of the interaction of the cutting edge of tillage implements with soil. Journal of Agricultural Engineering Research, 74(1), 91-101.

Godwin, R. J., \& Spoor, G. (1977). Soil failure with narrow tines. Journal of Agricultural Engineering Research, 22(3), 213-228.

Graham, J. (1968). Plane plastic failure in cohesionless soils. Geotechnique, 18(3), 301-316.

Grisso, R. D., Yasin, M., \& Kocher, M. F. (1996). Tillage implement forces operating in silty clay loam. Transactions of the ASAEAmerican Society of Agricultural Engineers, 39(6), 1977-1982.

Hettiaratchi, D. R. P., \& Reece, A. R. (1967). Symmetrical three-dimensional soil failure. Journal of Terramechanics, 4(3), 45-67.

Kawamura, H. (1985). Phase transition of the three-dimensional Heisenberg antiferromagnet on the layered-triangular lattice. Journal of the Physical Society of Japan, 54(9), 3220-3223.

Krastin, E. N. (1973). use of methods of theory of dimensional analysis for the evaluation of the draft characteristics of the plow during operation in different conditions. Dokfady Milsp, 8(1), 61-72.

McKyes, E, \& Ali, O. S. (1977). The cutting of soil by narrow blades. Journal of Terramechanics, 14(2), 43-58.

McKyes, Edward. (1985). Soil cutting and tillage. Elsevier.

Nicholson, R. I., Bashford, L. L., \& Mielke, L. N. (1984). Energy requirement for tillage from a reference implement. Paper-American Society of Agricultural Engineers [Microfiche Collection](USA). No. Fiche No. 84-1028.

O’callaghan, J. R., \& Farrelly, K. M. (1964). Cleavage of soil by tined implements. Journal of Agricultural Engineering Research, 9(3), 259-270.
Oni, K. C., Clark, S. J., \& Johnson, W. H. (1992). The effects of design on the draught of undercutter-sweep tillage tools. Soil and Tillage Research, 22(1-2), 117-130.

Onwualu, A. P., \& Watts, K. C. (1998). Draught and vertical forces obtained from dynamic soil cutting by plane tillage tools. Soil and Tillage Research, 48(4), 239-253.

Osman, M. S. (1964). The mechanics of soil cutting blades. Trans. of ASAE, 9(4), 318328 .

Payne, P. C. J. (1956). The relationship between the mechanical properties of soil and the performance of simple cultivation implements. Journal of Agricultural Engineering Research, 1(1), 23-50.

Perumpral, J. V, Grisso, R. D., \& Desai, C. S. (1983). A soil-tool model based on limit equilibrium analysis. Transactions of the ASAE, 26(4), 991-995.

Plouffe, C., Richard, M. J., Tessier, S., \& Lague, C. (1999). Validations of moldboard plow simulations with FEM on a clay soil. Transactions of the ASAE, 42(6), 1523.

Reaves, C. A., Cooper, A. W., \& Kummer, F. A. (1968). Similitude in performance studies of soil-chisel systems. Transactions of the ASAE, 11(5), 658-660.

Reece, A. R., \& Hettiaratchi, D. R. P. (1989). A slip-line method for estimating passive earth pressure. Journal of Agricultural Engineering Research, 42(1), 27-41.

Schafer, R. L., Young, S. C., Hendrick, J. G., \& Johnson, C. E. (1984). Control concepts for tillage systems. Soil and Tillage Research, 4(4), 313-320.

Swick, W. C., \& Perumpral, J. V. (1988). A model for predicting soil-tool interaction. Journal of Terramechanics, 25(1), 43-56.

Terzaghi, K. (1943). Theoretical soil mechanics. johnwiley \& sons. New York, 11-15.

Upadhyaya, S. K. (1984). Prediction of tillage implement draft. Microfiche Collection.

Yasin, M., Grisso, R., \& Bashford, L. (1991). Reference implement concept for predicting tillage draft. ASAE Paper No. 91-1525.

Yong, R. N., \& Hanna, A. W. (1977). Finite element analysis of plane soil cutting. Journal of Terramechanics, 14(3), 103125.

Young, D. F. (1968). Similitude of soil-machine systems. Transactions of the ASAE, 11(5), 
653-657.

Zein El-Din, A. M., \& Sayedahmed, A. A. (2000). A mathematical model for predicting draft forces for flat, chisel, sweep and winged chisel tools. M.J.Agric.Eng., 17(1), 208232.

Zein Eldin, A. M., Watts, K. C., Younis, S. M., \& Shaibon, M. (1990). Development of a two dimensional finite element model for soiltool interaction. Paper-American Society of
Agricultural Engineers, 90-1544.

Zhang, J.-W., Zhu, L., Chen, P., Wu, Q.-M., Wei, M., Yin, C.-L., \& Li, G.-L. (2020). Flowing interaction between cutting edge of ploughbreast with soil in shifting tillage operations. Engineering Applications of Computational Fluid Mechanics, 14(1), 1404-1415. 